\title{
Early High-contrast Imaging Results with Keck/NIRC2-PWFS: The SR 21 Disk
}

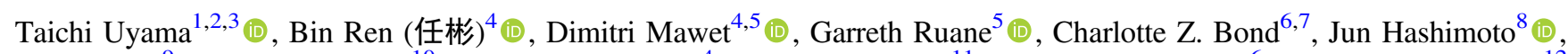

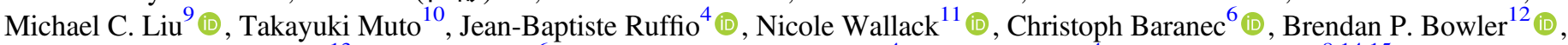 \\ Elodie Choquet ${ }^{13}$ (1) , Mark Chun ${ }^{6}$ (1) , Jacques-Robert Delorme $^{4}$, Kevin Fogarty ${ }^{4}$ (1) , Olivier Guyon ${ }^{8,14,15}$ (1),

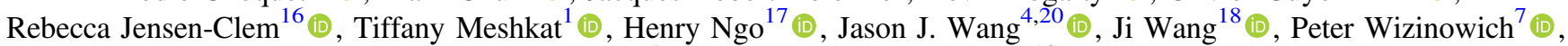 \\ Marie Ygouf $^{5}$ (10), and Benjamin Zuckerman ${ }^{19}$ (1) \\ ${ }^{1}$ Infrared Processing and Analysis Center, California Institute of Technology, 1200 E. California Blvd., Pasadena, CA 91125, USA \\ ${ }^{2}$ NASA Exoplanet Science Institute, Pasadena, CA 91125, USA \\ ${ }^{3}$ National Astronomical Observatory of Japan, 2-21-1 Osawa, Mitaka, Tokyo 181-8588, Japan \\ ${ }^{4}$ Department of Astronomy, California Institute of Technology, 1200 E. California Blvd., Pasadena, CA 91125, USA \\ 5 Jet Propulsion Laboratory, California Institute of Technology, 4800 Oak Grove Dr., Pasadena, CA, 91109, USA \\ ${ }_{7}^{6}$ Institute for Astronomy, University of Hawai'i at Mānoa, Hilo, HI 96720, USA \\ ${ }^{7}$ W.M. Keck Observatory, 65-1120 Mamalahoa Hwy., Kamuela, HI 96743, USA \\ ${ }^{8}$ Astrobiology Center of NINS, 2-21-1 Osawa, Mitaka, Tokyo 181-8588, Japan \\ ${ }^{9}$ Institute for Astronomy, University of Hawaii, 2680 Woodlawn Dr., Honolulu, HI 96822, USA \\ ${ }^{10}$ Division of Liberal Arts, Kogakuin University 2665-1, Nakano-cho, Hachioji-chi, Tokyo, 192-0015, Japan \\ ${ }^{11}$ Division of Geological \& Planetary Sciences, California Institute of Technology, Pasadena, CA 91125, USA \\ ${ }^{12}$ Department of Astronomy, The University of Texas at Austin, 2515 Speedway Blvd. Stop C1400, Austin, TX 78712, USA \\ ${ }^{13}$ Aix Marseille Univ, CNRS, CNES, LAM, Marseille, France \\ ${ }^{14}$ Subaru Telescope, National Astronomical Observatory of Japan, 650 North A'ohōkū Pl., Hilo, HI96720, USA \\ ${ }^{15}$ Steward Observatory, University of Arizona, Tucson, AZ 85721, USA \\ ${ }^{16}$ Astronomy \& Astrophysics Department, University of California, Santa Cluz, CA 95064, USA \\ ${ }^{17}$ NRC Herzberg Astronomy and Astrophysics, 5071 West Saanich Rd., Victoria, British Columbia, Canada \\ ${ }^{18}$ Department of Astronomy, The Ohio State University, 100 W. 18th Ave., Columbus, OH 43210, USA \\ ${ }^{19}$ Department of Physics \& Astronomy, University of California, 430 Portola Plaza, Los Angeles, CA 90095, USA \\ Received 2020 September 27; revised 2020 October 29; accepted 2020 October 30; published 2020 November 23
}

\begin{abstract}
High-contrast imaging of exoplanets and protoplanetary disks depends on wave front sensing and correction made by adaptive optics instruments. Classically, wave front sensing has been conducted at optical wavelengths, which made high-contrast imaging of red targets such as M-type stars or extincted T Tauri stars challenging. Keck/ NIRC2 has combined near-infrared (NIR) detector technology with the pyramid wave front sensor (PWFS). With this new module we observed SR 21, a young star that is brighter at NIR wavelengths than at optical wavelengths. Compared with the archival data of SR 21 taken with the optical wave front sensing we achieved $20 \%$ better Strehl ratio in similar natural seeing conditions. Further post-processing utilizing angular differential imaging and reference-star differential imaging confirmed the spiral feature reported by the Very Large Telescope/SpectroPolarimetric High-contrast Exoplanet REsearch instrument polarimetric observation, which is the first detection of the SR 21 spiral in total intensity at $L^{\prime}$ band. We also compared the contrast limit of our result $\left(10^{-4}\right.$ at 0.4 and $2 \times 10^{-5}$ at 1 !' 0 ) with the archival data that were taken with optical wave front sensing and confirmed the improvement, particularly at $\leqslant 0$ " 5 . Our observation demonstrates that the NIR PWFS improves AO performance and will provide more opportunities for red targets in the future.
\end{abstract}

Unified Astronomy Thesaurus concepts: Coronagraphic imaging (313); Protoplanetary disks (1300)

\section{Introduction}

High-contrast imaging has opened a new way of exploring and characterizing exoplanets around young stars (e.g., HR8799 bcde; Marois et al. 2008, 2010). This is achieved with large ground-based telescopes by correcting wave fronts of the incident light that are distorted by turbulence of the Earth's atmosphere. Adaptive optics (AO; Beckers 1993) provides real-time corrections to the wave front distortions using a guide star, which results in a point-spread function (PSF) close to a diffraction-limited pattern. Wave front sensing has classically been performed at optical wavelengths, in which case the visible brightness of the guide star is very important for the wave front sensing and correction. However, for some specific science targets such as M-type stars or extincted young stellar objects (YSOs) that are faint at optical wavelengths

\footnotetext{
${ }^{20} 51$ Pegasi b Fellow.
}

while bright in the near-infrared (NIR), wave front sensing at NIR wavelengths provides better AO performance. Recently, an NIR pyramid wave front sensor (PWFS) was installed in the AO system at Keck II (Bond et al. 2018, 2020) as part of the Keck Planet Imager and Characterizer (KPIC; Mawet et al. 2016). Science operations using the NIR-PWFS with Keck/ NIRC2 began in early 2019.

$\mathrm{EM}^{*}$ SR 21 (hereafter SR 21) is a young stellar object (YSO) in the Ophiuchus star-forming region with a distance of $138.4 \pm 1.1 \mathrm{pc}$ (Gaia Collaboration et al. 2018). Herczeg \& Hillenbrand (2014) found a spectral type of F7 and an age of $10 \mathrm{Myr}$ assuming $121 \mathrm{pc}$ for its distance. Sallum et al. (2019) inferred a spectral type of G3 for assuming the Gaia-based distance. Its spectral energy distribution (SED) shows strong infrared (IR) excess but lacks emission at $\sim 5 \mu \mathrm{m}$, which suggests a transitional disk with an inner gap (Brown et al. 2007). High-angular resolution studies have shown a large cavity (e.g., van der Marel et al. 2016), which together with 


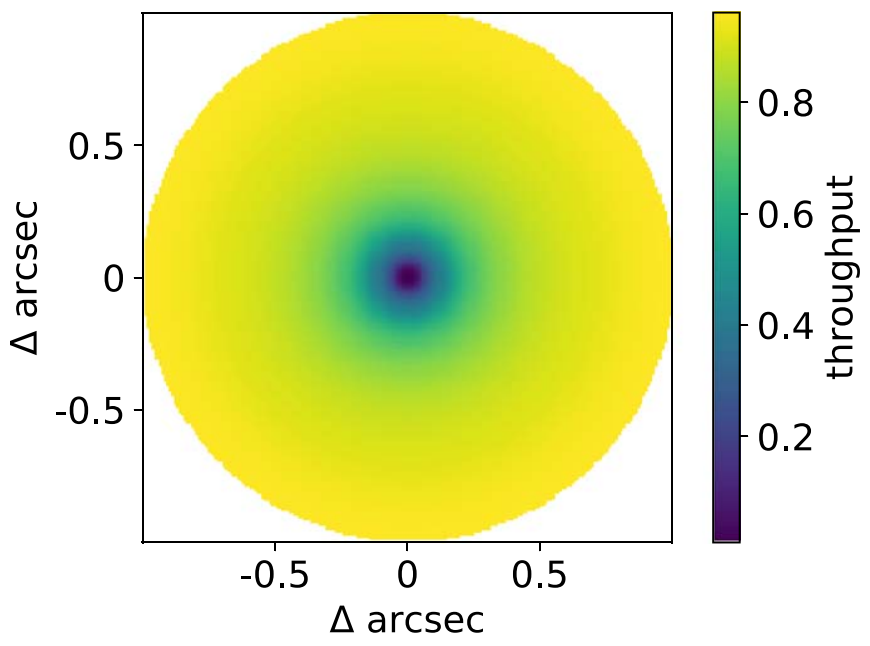

Figure 1. Simulated throughput map around the vortex mask. The off-axis SR 21 data used in this study were taken with an offset of $\sim 0$ " 28 from the vortex mask, which affected the throughput of the off-axis PSF.

complicated features such as gaps and spirals suggests possible planet formation within the disk (Sallum et al. 2019; MuroArena et al. 2020). This YSO is faint at optical wavelengths but bright at NIR wavelengths (e.g., $V=14.1, H=7.5$; Cutri et al. 2003; Rebull et al. 2018), making it a suitable testbed for high-contrast imaging with the PWFS to better explore its surrounding disk features and/or companions. In this study we present the latest observation of SR 21, which is among the earliest science targets of high-contrast imaging with the NIRPWFS following Wang et al. (2020), who observed PDS 70 using Keck/NIRC2 with the NIR-PWFS. Section 2 describes our observations, data reduction, and the results. Data analysis and discussion including the comparison with the archived Keck/NIRC2 data are described in Section 3. Finally our observations and results are summarized in Section 4.

\section{Observations and Results}

\subsection{Observations}

We observed SR 21 on 2020 May 31 UT with Keck/NIRC2 + PWFS in the $L^{\prime}$ band combined with the vector vortex coronagraph mask (inner working angle: IWA $\sim 100$ mas; Serabyn et al. 2017; Mawet et al. 2017) and angular differential imaging (ADI; Marois et al. 2006). The wave front sensing was carried out at the $H$ band. The total exposure time is $4110 \mathrm{sec}$ $(0.3 \mathrm{sec}$ per exposure $\times 100$ coadds $\times 137$ frames $)$ and the parallactic angle change is $56^{\circ} .31$. We also took an off-axis image offset by $\sim 0$ ". 28 from the vortex mask, which is comprised of 100 coadds each having an integration time of $0.0075 \mathrm{sec}$. Typical, the FWHM was measured to be 8.2 pixels (or 81.8 mas with a pixel scale of $9.972 \mathrm{mas} /$ pixel). We compared this unsaturated PSF with the simulated ideal PSF at the $L^{\prime}$ band, where we took into account throughput loss by the vortex mask (see Figure 1), and we obtained a Strehl ratio of $\sim 87 \%$ (approximately equivalent to the rms of the wave front errors $\simeq 200 \mathrm{~nm}$ ). The current throughput of the NIRC2-PWFS is $\sim 9 \%$ and the maximum elevation of SR 21 was $\sim 45^{\circ}$ during our observations, which affected the AO performance compared with the ideal case (Bond et al. 2018, 2020). The data were taken as a part of a shared-risk program using the NIRPWFS (PI: Dimitri Mawet) which was aimed at science verification with redder targets. In this program we did not observe suitable reference stars for reference-star differential imaging (RDI; Ruane et al. 2019) and thus we decided to use other data sets (see Table 1 and Section 2.2.1) that have the most comparable luminosity at the $H$ and $W 1$ bands to SR 21 among targets observed at this epoch as reference PSFs for the RDI reduction.

\subsection{Data Reduction and Results}

We first carried out pre-processing, including bad pixel correction, flat-fielding, sky subtraction, and image registration following Xuan et al. (2018) and Ruane et al. (2019). We then performed post-processing to subtract the stellar halo. We investigate two approaches of post-processing here: RDI to investigate the faint disk features and inner potential companions, and ADI to investigate outer potential companions. For both approaches, we used the pyKLIP package ${ }^{21}$ (Wang et al. 2015) that adopted the Karhunen-Loève Image Projection (KLIP; Amara \& Quanz 2012; Soummer et al. 2012) to produce the most likely reference PSF for the target exposure.

\subsubsection{Reference-star Differential Imaging}

As we did not take suitable data sets for the reference PSF, the RDI results can be affected by the quality of other stars in the reference library. In addition to the SR 21 data, we included WaOph 6 and WSB 52 data sets (PI: Charlotte Bond, Dimitri Mawet) in the PSF library (see Table 1 for the observing logs). We decided to use these two stars as reference stars because their $W 1$ magnitudes are the closest to SR 21 among all targets taken on and around 2020 May 31. These two stars are YSOs surrounded by protoplanetary disks, which have been resolved by Huang et al. (2018b, 2018a). The NIRC2 L'-band PSF for these targets may be influenced by the disks, which could impact the RDI reduction at particularly inner separations.

As mentioned above, the reference stars (WaOph 6 and WSB 52) used in this study are not ideal for the RDI reduction of SR 21 because (1) their $W 1$ magnitudes are not exactly the same, (2) observational conditions may vary from target to target, and (3) they possibly include the protoplanetary disk signals at the $L^{\prime}$ band, which can degrade the efficiency and output of the post-processing with RDI. We first conducted frame selection among the PSF library by taking advantage of the mean square error (MSE; Wang et al. 2004; Ruane et al. 2019). We evaluated MSE scores at separations $\leqslant 390$ mas $(\sim 5$ $\lambda / \mathrm{D})$ and removed the worst $20 \%$ of frames in each data set. After the frame selection the field of view (FoV) was cropped into 2 " $0 \times 2$ ". 0 , which is used to make the reference PSF. We performed RDI reduction including all the science data sets and the selected good reference data sets using pyKLIP.

Figure 2 shows the RDI-reduced result (Karhunen-Loèvethe number of basis vector; $\mathrm{KL}=5$ ) in total intensity, which is overlaid with contours from SPHERE/Infra-Red Dual Imaging and Spectrograph (IRDIS) $H$-band coronagraphic (left) and $J$-band noncoronagraphic (right) observations. We reduce the public SPHERE observations of SR 21 on UT 2018 March 1 (ESO GTO program 1100.C-0481(Q); PI: J.-L. Beuzit) using IRDAP (van Holstein et al. 2017, 2020). The bright extended feature at the position angle (PA) between $\sim 170^{\circ}$ and $\sim 270^{\circ}$ is clearly colocated with the spiral detected by SPHERE (Spiral 1 in Muro-Arena et al. 2020) and this detection is one of several

\footnotetext{
21 https://pyklip.readthedocs.io/en/latest/index.htmll
} 

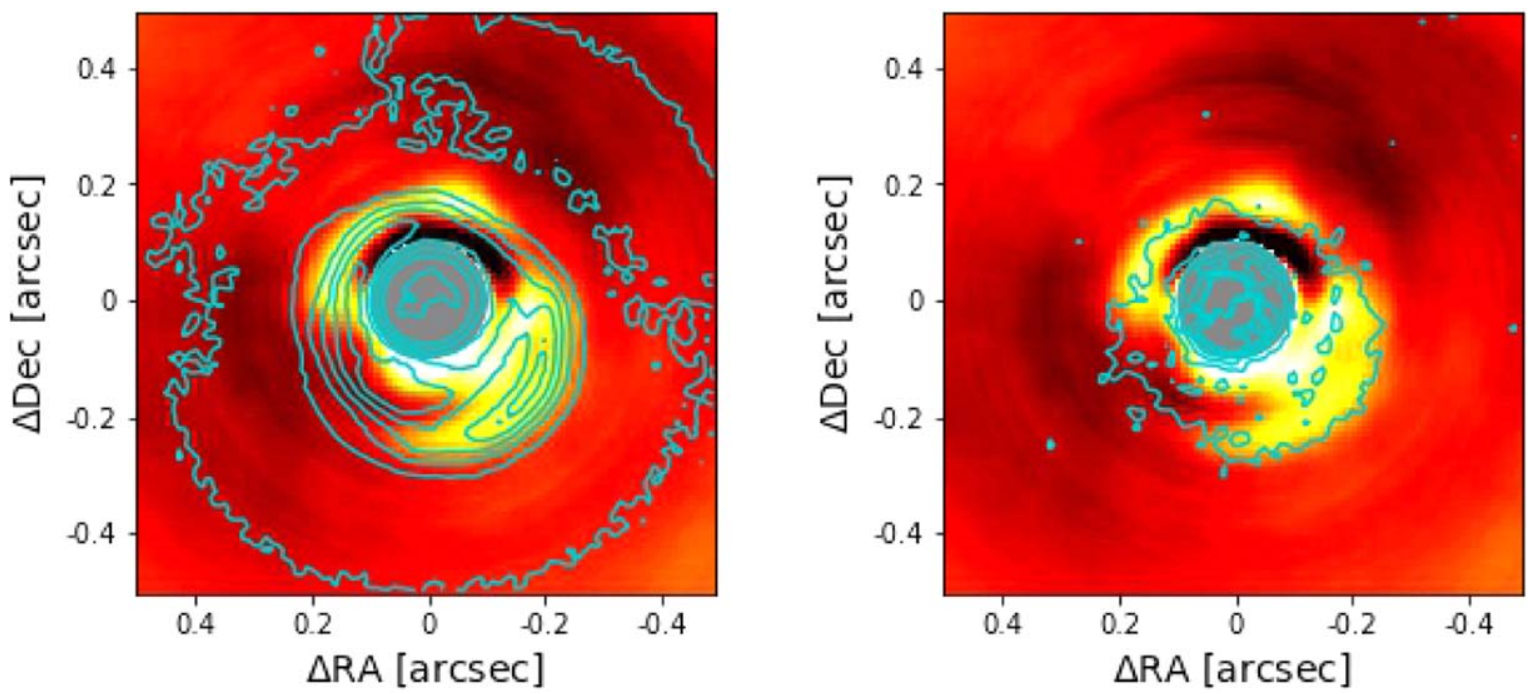

Figure 2. RDI-reduced image of SR $21(\mathrm{KL}=5)$ overlaid with SPHERE-PDI contours (cyan, left: $H$ band with coronagraph, right: $J$ band without coronagraph). The color scale is arbitrarily set to clearly show the spiral feature. The gray-masked area indicates the NIRC2 vortex coronagraph. North is up and east is left. The dark regions are due to oversubtraction by the RDI reduction, which is probably impacted by the imperfect background subtraction and/or the nonideal reference stars.

Table 1

Observing Log

\begin{tabular}{|c|c|c|c|c|c|}
\hline Target & Date [UT] & Total Exposure [s] & Seeing $\left[{ }^{\prime \prime}\right]^{\mathrm{a}}$ & $H[\mathrm{mag}]^{\mathrm{b}}$ & $W 1[\mathrm{mag}]$ \\
\hline SR 21 & 2020 May 31 & 4110 & 0.50 & 7.5 & 6.1 \\
\hline WaOph 6 & 2020 May 30 & 1230 & 0.49 & 7.6 & 7.6 \\
\hline WSB 52 & 2020 Jun 2 & 5040 & 0.47 & 9.2 & 7.5 \\
\hline
\end{tabular}

Notes.

${ }^{a}$ Mean DIMM seeing at the summit of Maunakea on each date. We note that during these observations part of the seeing information was not recorded. It is possible that the NIRC2 data were taken at different seeing conditions.

${ }^{\mathrm{b}}$ Two Micron All-Sky Survey photometry (Cutri et al. 2003).

${ }^{c}$ AllWISE photometry (Cutri et al. 2013).

cases that the spiral feature is clearly resolved in the $L^{\prime}$ band (e.g., HD 142527, HD 100546, MWC 758, and CQ Tau; Rameau et al. 2012; Currie et al. 2015; Reggiani et al. 2018; Uyama et al. 2020b). To test the potential contamination of the disk feature around the reference stars in the $L^{\prime}$ band, we checked the archival Very Large Telescope (VLT)/SpectroPolarimetric High-contrast Exoplanet REsearch instrument (SPHERE) $\mathrm{H}$-band polarimetric data set of WaOph6 taken on UT 2018 June 22 (ESO GTO program 1100.C-0481(Q); PI: J.-L. Beuzit). The SPHERE data set presents a very faint feature of the disk surface of $\mathrm{WaOpH} 6$, and the SPHEREbased SR 21 spiral arm is $\sim 30$ times brighter than the WaOph 6 disk at the same position, indicating that the contamination of the WaOph 6 disk on the NIRC2 PSF of SR 21 should be relatively very small. For WSB 52 there is no public SPHERE data in the VLT archive.

However, our observation did not confirm other features that the SPHERE observations reported. The inner area of the processed image has regions with both positive and negative intensity. Part of the positive areas might correspond to the real disk features but they are likely affected by the poor RDI reduction due to the nonideal reference stars.

\subsection{Angular Differential Imaging}

We conducted basic ADI reduction using pyKLIP using all of the 137 frames. We divided the NIRC2 FoV into 9 annuli $\times 4$ subsections to produce the most likely reference PSF at each area. We also assumed the real astrophysical signal, if any, moves 1 pixel from frame to frame to avoid heavy self-subtraction caused by the ADI reduction.

Figure 3 shows the ADI result $(\mathrm{KL}=20)$ for the larger FoV (left) and for the zoomed-in image (right) to compare with the RDI result. We did not detect any significant sources within $\sim 3^{\prime \prime}$. With a smaller number of KL we also see the spiral feature in the ADI-reduced image (Figure 4). In the zoomed-in image of Figure 3, there are two marginal point-like sources to the southwest and south that are coincident with the spiral feature and stable among KLs between 10 and 30. However, their signal-to-noise ratios $(\mathrm{S} / \mathrm{Ns})$ are not large enough to robustly claim a detection $(\sim 3.5,3.7$, respectively). There is another marginal source outside the spiral feature at the southeast but this is fainter than the sources mentioned above. In this study, we regard them as the residuals of the spiral feature or uncorrected speckle noise and future observation that can explore deeper. Contrast at $\sim 0$ !' $2-0$ ". 3 will help to identify the nature of these sources especially in the context of planetdisk interactions in the SR 21 system. We also injected fake sources at separations ranging from 0 ". $1-3$ ". 0 and at a variety of PAs to test whether the inject sources are reliably reproduced after the ADI reduction. We altered the contrast levels of the fake sources as a function of radius $\left(7.5 \times 10^{-4}\right.$ at $r \leqslant 0$ !" 3 , $1.5 \times 10^{-4}$ at $0 ! 3<r \leqslant 0 ! 7$, and $5.0 \times 10^{-5}$ at $r>0$ !'7) 

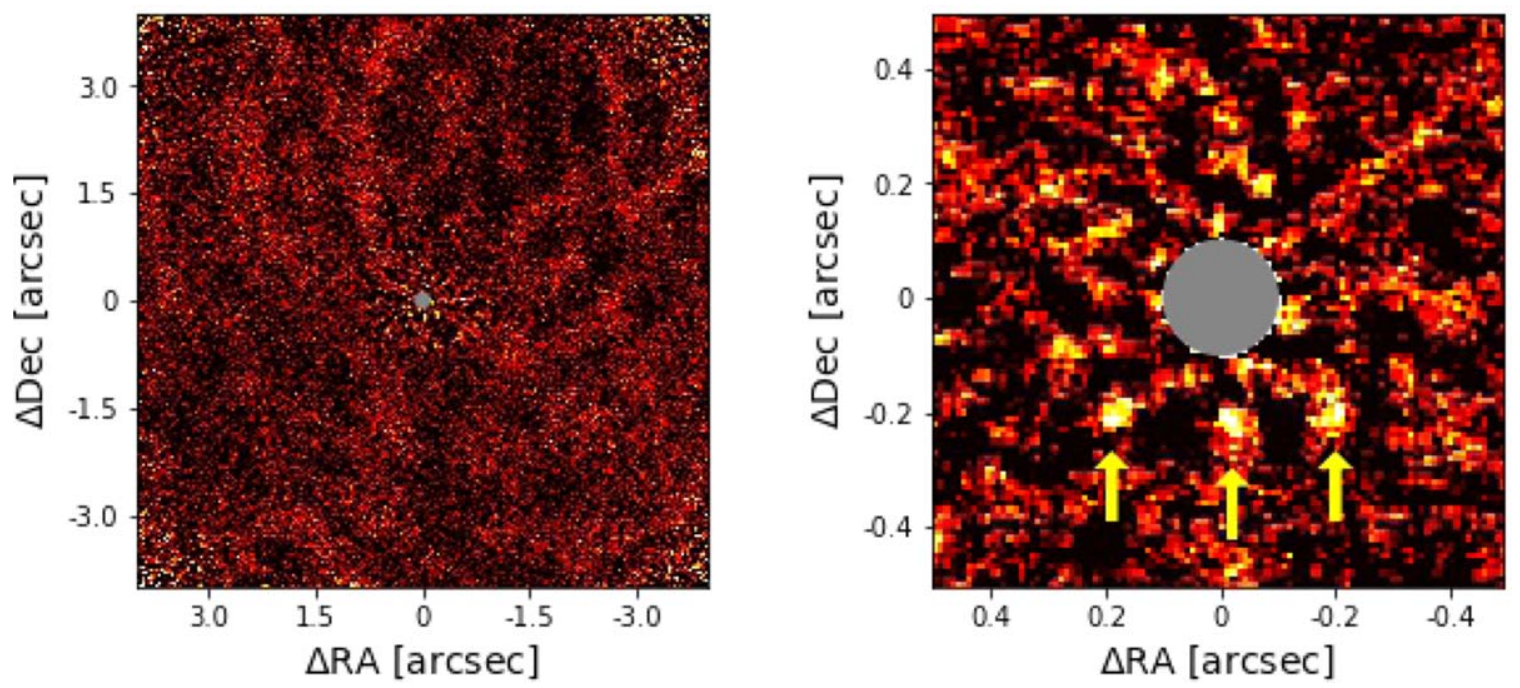

Figure 3. ADI-reduced image of SR 21 ( $\mathrm{KL}=20$, left: larger FoV, right: zoomed-in image). The marginal point sources are indicated by yellow arrows (see also Section 2.3). The southwest and south (right and middle) sources coincide with the spiral feature. The gray-masked area indicates the NIRC2 vortex coronagraph. North is up and east is left.
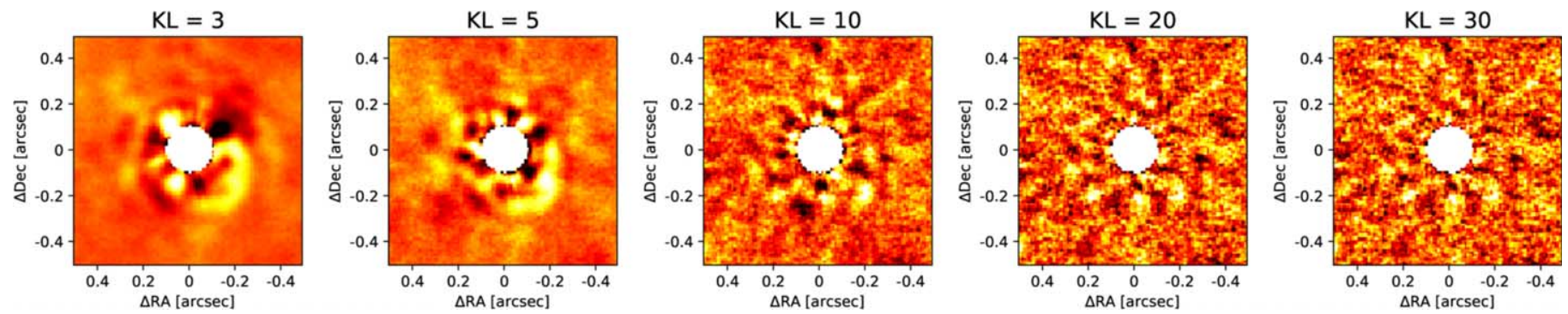

Figure 4. Comparison of the ADI result at different KLs. The small-KL image $(\mathrm{KL}<10)$ is more sensitive to an extended feature such as the spiral while the large $\mathrm{KL}$ is more sensitive to a point source.
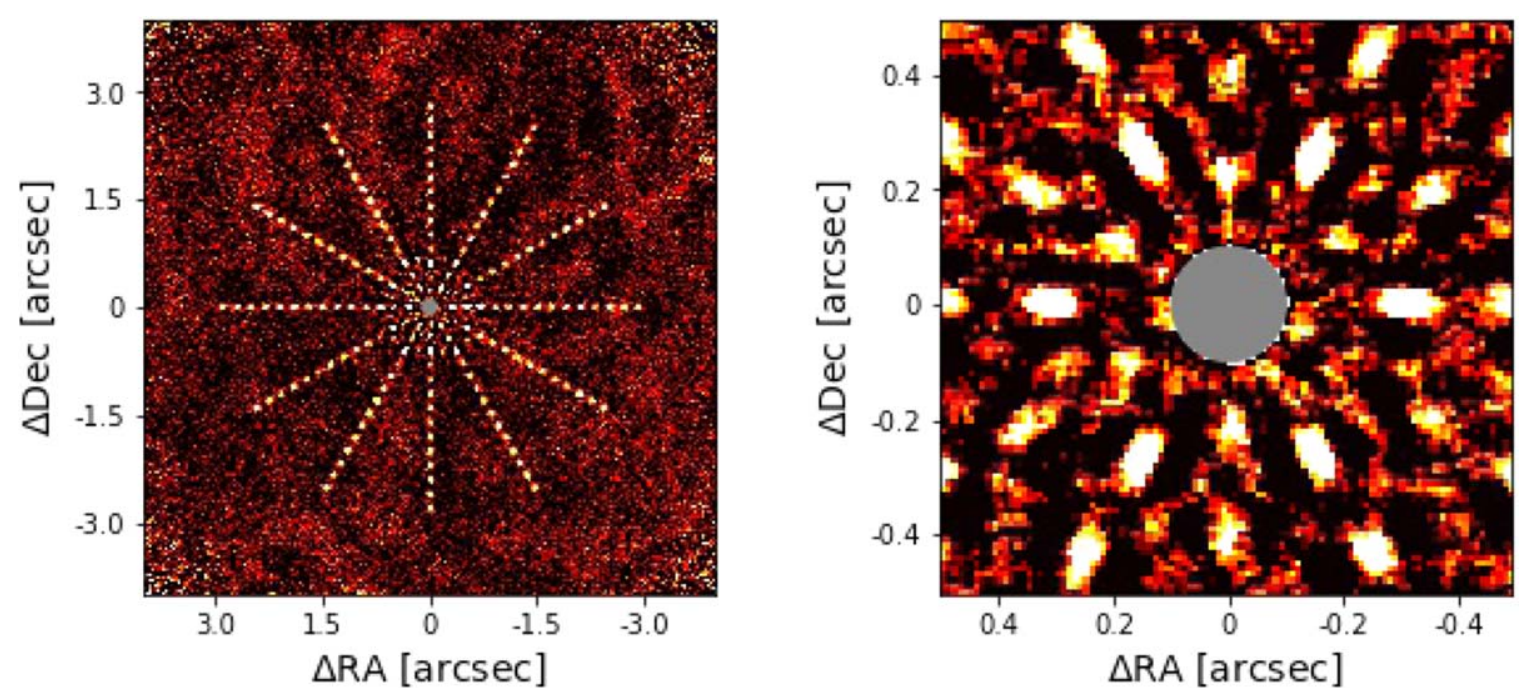

Figure 5. Same as Figure 3 with injected fake sources at separations ranging from 0 " $1-3$ !" 0 . The radially elongated shapes of the fake sources are affected by selfsubtraction in the ADI reduction along the azimuthal direction.

because a bright source can result in large flux loss. Figure 5 shows a result of the injection test. Although part of the disk features may affect the outcome of the ADI reduction, the injection test shows that the point sources can be reliably recovered.

\section{Discussion}

\subsection{Spiral Feature}

The disk feature detected in the $L^{\prime}$ band may be explained by two scenarios: scattering or self-luminosity. If the spiral feature 
is self-luminous it may be a result of heating a shock around the protoplanet, which would indicate planet-disk interactions (e.g., Lyra et al. 2016).

We use our RDI result to extract the surface brightness of the spiral feature because RDI causes only modest signal loss (e.g., $\lesssim 15 \%$ with the principal component analysis (PCA)-based RDI reduction of the disk feature; Uyama et al. 2020a) compared with self-subtraction of ADI, particularly at such small separations. We converted the pixel count into surface brightness by comparing the photometric result of the off-axis PSF (aperture radius $=3.5 \times$ FWHM) with the Spitzer/ IRAC-3.6 $\mu \mathrm{m}$ value $(1.23 \pm 0.01 \mathrm{Jy}$; Gutermuth et al. 2009). We traced the spiral feature and investigated several areas, which measures $\sim 60,100,80,70 \mathrm{mJy} \operatorname{arcsec}^{-2}$ at PA $=180^{\circ}$, $210^{\circ}, 240^{\circ}$, and $270^{\circ}$, respectively. ${ }^{22}$ Assuming these values reflect thermal emissions from the optically thick surface of the spiral, the typical temperature of the spiral corresponds to $\sim 200 \mathrm{~K}$. On the other hand, assuming $L_{\mathrm{SR} 21}=11 L_{\odot}$ (Francis \& van der Marel 2020) and 0!'23 for the typical separation of the spiral, the effective temperature at the spiral is estimated at $\lesssim 80 \mathrm{~K}$ and the derived temperature from our RDI result suggests the scattering effect or another heating mechanism (e.g., the shock scenario; Lyra et al. 2016).

We also calibrated the SPHERE $H$-band $\mathrm{Q}_{\phi}$ image following the IRDAP pipeline. We measured that the polarimetric intensity at the spiral surface is $35-40 \mathrm{mJy}^{-2} \operatorname{arsec}^{-2}$ along the spine, with an error of $\pm 1.3 \mathrm{mJy} \mathrm{arcsec}{ }^{-2}$, which is defined as the standard deviation in an annular area (at radii between 175 and 225 mas) of the calibrated SPHERE $\mathrm{U}_{\phi}$ image that is smoothed with a Gaussian kernel ( $\sigma=2$ pixel). By comparing these values with radiative transfer simulations one can infer the scattering characteristics and the possibility of thermal emission at the spiral surface, but we note that simply comparing our $L^{\prime}$-band total intensity with the SPHERE $H$-band polarimetric imaging result leaves uncertainty on polarization ratio. Obtaining total intensity and polarization intensity at the same wavelength is useful to characterize the scattering mechanisms at the disk surface in detail.

As mentioned in Section 2, the reference stars we used in this study are not suitable for SR 21 and thus the extracted surface brightness may be biased. Future follow-up observations with more suitable reference stars will help to better investigate the whole disk feature including the spiral in the $L^{\prime}$ band.

\subsection{Detection Limits}

We convolved the ADI-reduced image by a circular aperture with a radius of FWHM/2 and then calculated the radial noise profile, which is then compared with the unsaturated PSF of SR 21 . We calibrated this radial profile by the flux loss ratio at each separation estimated from the injection test result. Figure 6 shows our $5 \sigma$ contrast limit: we achieved $10^{-4}$ at 0 " 4 and $2 \times 10^{-5}$ at 1 "'0. With the COND03 model (Baraffe et al. 2003) assuming $10 \mathrm{Myr}$ overlaid in Figure 6, we set a constraint on $\sim 16 M_{\text {Jup }}, 10 M_{\text {Jup }}$, and $5 M_{\text {Jup }}$ at $\sim 40$ au, $55 \mathrm{au}$, and $110 \mathrm{au}$, respectively. We assumed $5.8 \mathrm{mag}$ at the $L^{\prime}$ band, which is different from Muro-Arena et al. (2020). Their assumption of $6.8 \mathrm{mag}$ is equivalent to $0.48 \mathrm{Jy}$ at $3.8 \mu \mathrm{m}$ and this value is fainter than the photometric values at adjacent

\footnotetext{
${ }^{22}$ We do not present the error bars for these values because we are not able to evaluate systematic errors caused by the nonideal reference stars. The background noise estimated from the standard deviation in an annular area at $\sim 1{ }^{\prime \prime} 0$ in the RDI-reduced image corresponds to $6 \mathrm{mJy} \operatorname{arcsec}^{-2}$.
}

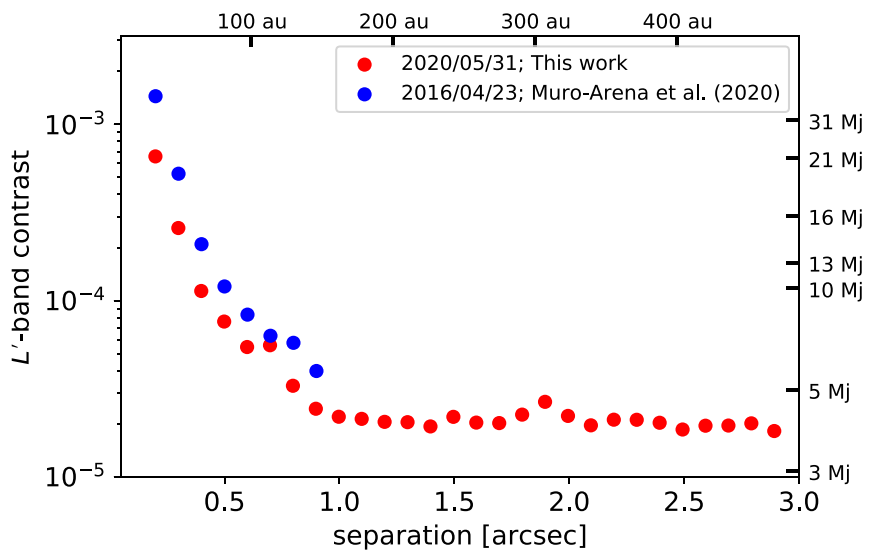

Figure 6. $5 \sigma$ contrast limits of the ADI $(\mathrm{KL}=20)$ result of our data (red) compared with approximate values of the archival data (blue). We adopted $L^{\prime}=5.8 \mathrm{mag}$, which is different from Muro-Arena et al. (2020), for the SR 21 luminosity. For the plot of the previous data we checked the contrast limit presented in Figure A.1 of Muro-Arena et al. (2020) and selected several points between 0 !" $2-0$ ! 9 .

wavelengths (e.g., $\sim 1.1-1.3 \mathrm{Jy}$ at 3.5-4.6 $\mu \mathrm{m}$; Gutermuth et al. 2009; Cutri et al. 2013; Schlafly et al. 2019). Our assumption of $5.8 \mathrm{mag}$ is equivalent to the Spitzer/IRAC-3.6 $\mu \mathrm{m}$ value. Our mass limit is consistent with an updated mass limit of the archival data from Figure A.1 of Muro-Arena et al. (2020). We also took into account extinction by the interstellar medium considering $A_{V}=6.2$ (Herczeg \& Hillenbrand 2014) and the wavelength dependence of extinction $\left(A_{\lambda} \propto \lambda^{-1.75}\right.$ : Draine 1989), which corresponds to $A_{L^{\prime}}=0.2$.

The evolutionary model we adopted in Figure 6 does not include the accreting mechanism. Given that the gas in the SR 21 disk has not been fully depleted at the predicted location the potential protoplanet would likely be actively accreting. So we also compare our contrast limits with a circumplanetary disk model (Zhu 2015), with which the $L^{\prime}$-band luminosity is calculated as a function of the product of planet mass $(M)$ and accretion rate $(M \dot{M})$ and inner radius of the circumplanetary disk $\left(R_{\mathrm{in}}\right)$. We follow Ruane et al. (2017) who investigated constraints on the potential accreting planets in the TW Hya disk. Muro-Arena et al. (2020) predicted a $\lesssim 1 M_{\text {Jup }}$ protoplanet at 44 au and PA $\sim 11^{\circ}$ and we use our contrast limit to set a constraint on the mass accretion rate onto this potential protoplanet. Figure 7 shows the comparison between our contrast limit at 0 ". 3 (contrast: $2.6 \times 10^{-4}$ ) and the cirucmplanetary disk model at a given inner radii $\left(1 R_{\mathrm{Jup}} \leqslant R_{\text {in }} \leqslant\right.$ $\left.4 R_{\text {Jup }}\right)$. Even if we assume $1 M_{\text {Jup }}$ for the potential protoplanet as predicted in Muro-Arena et al. (2020), we are not able to solve the degeneracy between the mass accretion rate and the inner radius of the circumplanetary disk because no observational evidence of the circumplanetary disk in the SR 21 disk has been reported. Therefore our detection limit could set an upper limit of the accretion rate to $\dot{M} \lesssim 1.2 \times 10^{-5} M_{\text {Jup }} \mathrm{yr}^{-1}$ by referring to a $R_{\text {in }}=4 R_{\mathrm{Jup}}$ case in the circumplanetary disk model.

These estimations of the mass/accretion rate limits do not include potential extinction of the SR 21 disk itself. This leaves uncertainty on the mass estimation but this may not be as important because the $L^{\prime}$-band detection limits are less subject to the extinction than shorter NIR wavelengths (e.g., JHK bands). 


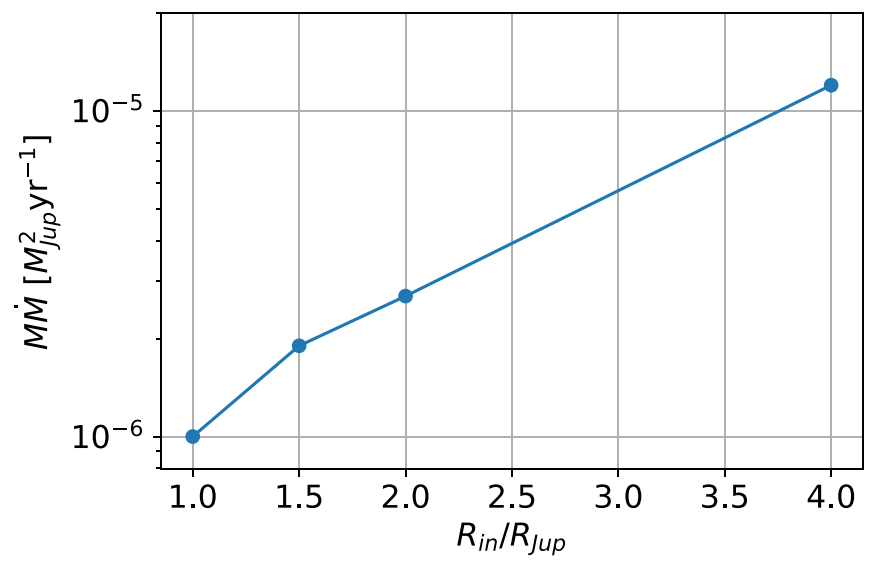

Figure 7. Comparison of our detection limits with the circumplanetary disk model (Zhu 2015) at 44 au ( 0." 3) where Muro-Arena et al. (2020) predicted a $\lesssim 1 M_{\text {Jup }}$ planet. Assuming $\sim 1 M_{\text {Jup }}$ for the mass the vertical axis (the product of mass $M$ and mass accretion rate $\dot{M}$ ) is interpreted as the accretion rate and thus this comparison can be used to constrain the accretion rate. The horizontal axis corresponds to inner radius of the circumplanetary disk $\left(R_{\mathrm{in}}\right)$.

\subsection{Comparison with the Archival Data}

Compared with the archival Keck/NIRC2 $L^{\prime}$-band data taken on 2016 April 23 (PI: Van der Marel; see Appendix A of Muro-Arena et al. 2020), we detected the spiral feature in both of the RDI (Figure 2) and ADI (KL $=3$ and 5 at Figure 4) reductions, while Muro-Arena et al. (2020) did not report any disk features in the archival Keck data. Our detection limit also achieves a better contrast level particularly at inner separations than the archival data (see Figure 6 for the comparison of the detection limits). At separations $\geqslant 0$ ". 5 the difference of the contrast limits is likely related to the difference in the exposure time (4110 sec and $2160 \mathrm{sec}$ for our observation and the previous observation, respectively). We also note that the difference of field rotation (56.31 and $18^{\circ} .19$, respectively) may also affect the detection limits at inner separations because a ratio of flux loss made by the ADI reduction can be affected by the rotation angle.

The main difference is that we used the vortex coronagraph and NIR-PWFS while the archival data were taken with the optical wave front sensing and no coronagraph mask. We took into account the effect of the vortex mask when we measured the Strehl ratio (see Section 2.1). The observing conditions are also differenttypical DIMM seeings were 0".50 and 0."77 on 2020 May 31 and 2016 April 23 respectively. We measured the Strehl ratio of the archival data to be $\sim 69 \%$, which is smaller than $\sim 87 \%$ that we measured for our observation. Although seeing was slightly larger on 2016 April 23 when the archival data were taken, the majority of the additional wave front errors due to the increased seeing should be outside the AO correction radius. The increase of the Strehl ratio by $\sim 20 \%$ is likely produced by the NIR-PWFS suggesting that the NIR-PWFS meaningfully improved the AO performance of Keck/NIRC2 on such a red target.

\section{Summary}

We have presented the latest Keck/NIRC2 $L^{\prime}$-band observation of SR 21. The observation was conducted with an NIR $(H$ band) PWFS and the Strehl ratio is measured to be $\sim 87 \%$ (approximately $200 \mathrm{~nm}$ for rms of the wave front errors). We used the PyKLIP package to post-process the observation utilizing RDI and ADI techniques. In the RDI-reduced image, we confirmed the spiral feature in total intensity at the $L^{\prime}$ band, which is consistent with the VLT/SPHERE polarimetric observation. However, we did not observe suitable reference stars for SR 21 but instead used WaOph 6 and WSB 52, which might also include disk signals. Therefore our RDI result may have been affected by these nonideal reference stars. Our ADI reduction did not detect any convincing companion candidates but we confirmed the spiral feature at small KLs $(\mathrm{KL} \leqslant 5)$. In addition to the spiral feature at small KLs, we see two pointlike sources with $\mathrm{S} / \mathrm{Ns}$ of $\sim 3.5-3.7$ at $\mathrm{KL}=10-30$ but they may be residuals of the spiral feature. We calculated a $5 \sigma$ contrast limit from the ADI result, which achieved $10^{-4}$ at 0.4 and $2 \times 10^{-5}$ at 1 !' 0 . Assuming the COND03 model and $10 \mathrm{Myr}$, we converted the contrast limit into a mass limit-16 $M_{\text {Jup }}$, $10 M_{\text {Jup }}$, and $5 M_{\text {Jup }}$ at $40 \mathrm{au}$, $50 \mathrm{au}$, and $110 \mathrm{au}$, respectively. Compared with the archival data taken with optical wave front sensing we achieved the better Strehl ratio and better contrast level, which suggests that the NIR PWFS improved the AO performance of Keck/NIRC2 and will provide more opportunities for red targets. As the spiral feature is affected by the bias of the reduction method and the reference stars used in this study, future observation of this system will provide better RDI results and enable a detailed discussion on the surface brightness distribution of the protoplanetary disk.

The authors would like to thank the anonymous referee for the constructive comments to improve the clarity of the manuscript. We thank Christian Ginski for sharing the updated detection limit of the archival Keck/NIRC2 data. Some of the data presented herein were obtained at the W. M. Keck Observatory, which is operated as a scientific partnership among the California Institute of Technology, the University of California, and the National Aeronautics and Space Administration. The Observatory was made possible by the generous financial support of the W. M. Keck Foundation. Based on observations collected at the European Organisation for Astronomical Research in the Southern Hemisphere under ESO program 1100.C-0481(Q). This research has made use of NASA's Astrophysics Data System Bibliographic Services. This research has made use of the SIMBAD database (Wenger et al. 2000), operated at CDS, Strasbourg, France. This research has made use of the VizieR catalog access tool, CDS, Strasbourg, France (doi:10.26093/cds/vizier). The original description of the VizieR service was published in Ochsenbein et al. (2000).

T.U. acknowledges JSPS overseas research fellowship. M.L. acknowledges funding from the National Science Foundation under grants AST-1518339. The Keck infrared pyramid wave front sensor was developed with support from the National Science Foundation under grants AST-1611623 and AST1106391, as well as the Heising Simons Foundation under the Keck Planet Imager and Characterizer project. This research is partially supported by NASA ROSES XRP, award 80NSSC19K0294. Part of this work was carried out at the Jet Propulsion Laboratory, California Institute of Technology, under contract with the National Aeronautics and Space Administration (NASA).

We wish to acknowledge the critical importance of the current and recent Maunakea Observatories day crew, 
technicians, telescope operators, computer support, and office staff employees, especially during the challenging times presented by the COVID-19 pandemic. Their expertise, ingenuity, and dedication is indispensable to the continued successful operation of these observatories. The authors wish to acknowledge the very significant cultural role and reverence that the summit of Maunakea has always had within the indigenous Hawaiian community. We are most fortunate to have the opportunity to conduct observations from this mountain.

Facility: Keck:II (NIRC2).

Software: pyKLIP (Wang et al. 2015), IRDAP (van Holstein et al. 2017, 2020).

\section{ORCID iDs}

Taichi Uyama (ib https://orcid.org/0000-0002-6879-3030 Bin Ren (任涁) (iD https://orcid.org/0000-0003-1698-9696 Dimitri Mawet (iD https://orcid.org/0000-0002-8895-4735 Garreth Ruane (i) https://orcid.org/0000-0003-4769-1665 Jun Hashimoto (i) https://orcid.org/0000-0002-3053-3575 Michael C. Liu (ID https://orcid.org/0000-0003-2232-7664 Jean-Baptiste Ruffio (iD https://orcid.org/0000-00032233-4821

Nicole Wallack (D) https://orcid.org/0000-0003-0354-0187 Christoph Baranec (i) https://orcid.org/0000-0002-1917-9157 Brendan P. Bowler (iD https://orcid.org/0000-0003-2649-2288 Elodie Choquet (i) https://orcid.org/0000-0002-9173-0740 Mark Chun (iD https://orcid.org/0000-0002-8462-0703 Kevin Fogarty (iD https://orcid.org/0000-0002-2691-2476 Olivier Guyon (ib https://orcid.org/0000-0002-1097-9908 Rebecca Jensen-Clem (1) https://orcid.org/0000-00030054-2953

Tiffany Meshkat (iD https://orcid.org/0000-0001-6126-2467

Henry Ngo (i) https://orcid.org/0000-0001-5172-4859

Jason J. Wang (i) https://orcid.org/0000-0003-0774-6502

Ji Wang (iD https://orcid.org/0000-0002-4361-8885

Peter Wizinowich (iD https://orcid.org/0000-0002-1646-442X

Marie Ygouf (iD https://orcid.org/0000-0001-7591-2731

Benjamin Zuckerman (ib https://orcid.org/0000-0001-

6809-3045

\section{References}

Amara, A., \& Quanz, S. P. 2012, MNRAS, 427, 948

Baraffe, I., Chabrier, G., Barman, T. S., et al. 2003, A\&A, 402, 701 Beckers, J. M. 1993, ARA\&A, 31, 13

Bond, C. Z., Cetre, S., Lilley, S., et al. 2020, JATIS, 6, 039003

Bond, C. Z., Wizinowich, P., Chun, M., et al. 2018, Proc. SPIE, 10703, 107031 Z Brown, J. M., Blake, G. A., Dullemond, C. P., et al. 2007, ApJL, 664, L107

Currie, T., Cloutier, R., Brittain, S., et al. 2015, ApJL, 814, L27

Cutri, R. M., Skrutskie, M. F., van Dyk, S., et al. 2003, yCat, 2246, 0

Cutri, R. M., Wright, E. L., Conrow, T., et al. 2013, yCat, 2328, 0

Draine, B. T. 1989, ESASP, 22, 93

Francis, L., \& van der Marel, N. 2020, ApJ, 892, 111

Gaia Collaboration, Brown, A. G. A., Vallenari, A., et al. 2018, A\&A, 616, A1 Gutermuth, R. A., Megeath, S. T., Myers, P. C., et al. 2009, ApJS, 184, 18 Herczeg, G. J., \& Hillenbrand, L. A. 2014, ApJ, 786, 97

Huang, J., Andrews, S. M., Dullemond, C. P., et al. 2018a, ApJL, 869, L42

Huang, J., Andrews, S. M., Pérez, L. M., et al. 2018b, ApJL, 869, L43

Lyra, W., Richert, A. J. W., Boley, A., et al. 2016, ApJ, 817, 102

Marois, C., Lafrenière, D., Doyon, R., et al. 2006, ApJ, 641, 556

Marois, C., Macintosh, B., Barman, T., et al. 2008, Sci, 322, 1348

Marois, C., Zuckerman, B., Konopacky, Q. M., et al. 2010, Natur, 468, 1080

Mawet, D., Choquet, É., Absil, O., et al. 2017, AJ, 153, 44

Mawet, D., Wizinowich, P., Dekany, R., et al. 2016, Proc. SPIE, 9909, 99090D

Muro-Arena, G. A., Ginski, C., Dominik, C., et al. 2020, A\&A, 636, L4

Ochsenbein, F., Bauer, P., \& Marcout, J. 2000, A\&AS, 143, 23

Rameau, J., Chauvin, G., Lagrange, A. M., et al. 2012, A\&A, 546, A24

Rebull, L. M., Stauffer, J. R., Cody, A. M., et al. 2018, AJ, 155, 196

Reggiani, M., Christiaens, V., Absil, O., et al. 2018, A\&A, 611, A74

Ruane, G., Mawet, D., Kastner, J., et al. 2017, AJ, 154, 73

Ruane, G., Ngo, H., Mawet, D., et al. 2019, AJ, 157, 118

Sallum, S., Skemer, A. J., Eisner, J. A., et al. 2019, ApJ, 883, 100

Schlafly, E. F., Meisner, A. M., \& Green, G. M. 2019, ApJS, 240, 30

Serabyn, E., Huby, E., Matthews, K., et al. 2017, AJ, 153, 43

Soummer, R., Pueyo, L., \& Larkin, J. 2012, ApJL, 755, L28

Uyama, T., Currie, T., Christiaens, V., et al. 2020a, ApJ, 900, 135

Uyama, T., Muto, T., Mawet, D., et al. 2020b, AJ, 159, 118

van der Marel, N., van Dishoeck, E. F., Bruderer, S., et al. 2016, A\&A, 585, A58

van Holstein, R. G., Girard, J. H., de Boer, J., et al. 2020, A\&A, 633, A64

van Holstein, R. G., Snik, F., Girard, J. H., et al. 2017, Proc. SPIE, 10400, 1040015

Wang, J. J., Ginzburg, S., Ren, B., et al. 2020, AJ, 159, 263

Wang, J. J., Ruffio, J.-B., de Rosa, R. J., et al. 2015, pyKLIP: PSF Subtraction for Exoplanets and Disks, Astrophysics Source Code Library, ascl:1506.001

Wang, Z., Bovik, A. C., Sheikh, H. R., \& Simoncelli, E. P. 2004, ITIP, 13, 600

Wenger, M., Ochsenbein, F., Egret, D., et al. 2000, A\&AS, 143, 9

Xuan, W. J., Mawet, D., Ngo, H., et al. 2018, AJ, 156, 156

Zhu, Z. 2015, ApJ, 799, 16 